\section{A One-pass Semi-quantitative Method for Extraction and Analysis of Carotenoids and Tocopherols in Carrot}

\author{
Thomas C. Koch and Irwin L. Goldman ${ }^{1}$ \\ Department of Horticulture, University of Wisconsin-Madison, 1575 Linden \\ Drive, Madison, WI 53706
}

Additional index words. HPLC, vitamin A, vitamin E, lycopene, $\alpha$-carotene, $\beta$-carotene, $\alpha$-tocopherol, Daucus carota

\begin{abstract}
Carotenoids and tocopherols are health-functional phytochemicals that occur in a wide range of fruit and vegetable crops. These two classes of compounds are synthesized from a common precursor, geranyl-geranyl pyrophosphate, and are typically analyzed separately via high-performance liquid chromatography (HPLC) techniques. Because carotenoids and tocopherols are present in many edible horticultural crops, it would be advantageous to measure them simultaneously in plant tissues. Herein we report a one-pass reverse-phase HPLC method for extraction and analysis of carotenoids and tocopherols in carrot that can be extended to other high-moisture plant organs. Elution times ranged from 5 minutes for $\alpha$-tocopherol to 24 minutes for $\beta$-carotene. This method improves the efficiency of analyzing these compounds by up to $50 \%$, and should increase the efficiency of assessing carotenoid and tocopherol profiles in horticultural crops.
\end{abstract}

Carotenoids and tocopherols are of great interest in the health functionality of vegetable crops. Carotenoids are antioxidants integral to all photosynthetic organisms, preventing formation of reactive oxygen radicals produced during photosynthesis and aiding in light harvest by chloroplast photosystems $(\mathrm{Bu}-$ chanan et al., 2000). Their bright colors attract pollinators and other organisms (Buchanan et al., 2000). Carotenoids are comprised of long isoprenoid chains that serve as the source of provitamin $\mathrm{A}$, an essential micronutrient in the human diet. Their antioxidant may help prevent macular degeneration (Ito et al., 1999), atherosclerosis (Jacques, 1999) and cancer (Prakash et al., 2001). Tocopherols, a related class of antioxidants, are important in maintaining membrane integrity through protection from lipid oxidation (Grusak and DellaPenna, 1999). Tocopherols possess a hydrophobic tail which associates with membrane lipids and an aromatic ring that helps control reactive oxygen species, preventing oxidation of surrounding tissue (Grusak and DellaPenna, 1999). The four isomers of tocopherols $(\alpha$, $\beta, \gamma, \delta$ ) serve as vitamin $\mathrm{E}$, with $\alpha$-tocopherol showing the highest amount of bioactivity in humans (Hirschberg, 1999). Both carotenoids and tocopherols are synthesized from a common precursor, geranylgeranyl-pyrophosphate (Hirschberg, 1999). They have been most accurately measured using liquid chromatography techniques.

\section{Materials and Methods}

We have developed a one-pass method for extraction and analysis of carotenoids and

Received for publication: 7 Mar. 2003. Accepted for publication 3 June 2003.

${ }^{1}$ To who reprint requests should be addressed; e-mail ilgoldma@wisc.edu. tocopherols in carrot. Our method allows for ease of detection of these compounds, facilitating a more rapid increase through selecting them simultaneously. Most tocopherol extraction protocols have been developed for seed oils (Emmons et al., 1999; Psomiadou et al., 2000; Tadmor et al., 2000). Our technique, which is based on Grela et al. (1999), allows for detection of both classes of compounds in high moisture plant tissue such as fruits and vegetables. Results are reported for the xylem and phloem of carrot roots, although the technique has also successfully been used for assaying carotenoids and tocopherols in carrot leaf tissue and tomato fruits.

Carrot accessions were a random sample of current processing and fresh market commercial hybrids. Accessions were planted in a randomized complete-block design present in orange carrot roots. consisting of four replications on muck soil ( $>20 \%$ organic matter) in Randolph, Wisc., during the 2001 growing season. At 102 d after planting, xylem and phloem samples were collected from the upper third of the carrot root, with about equal amounts bulked from each of 10 roots within each accession. Samples were lyophilized and ground using a pre-chilled mortar and pestle under low light conditions. Samples were stored at $-20^{\circ} \mathrm{C}$ in 15 -mL screw-top polypropylene centrifuge tubes until extraction.

An aliquot of $200 \mathrm{mg}$ accurately weighed to the nearest $\mathrm{mg}$ was removed from each sample and extracted in $2.0 \mathrm{~mL}$ heptane containing $0.05 \%$ butylated hydroxytoluene (BHT). Samples were vortexed for $2 \mathrm{~min}$ and centrifuged at $3000 g_{\mathrm{n}}$ at $0{ }^{\circ} \mathrm{C}$ for $10 \mathrm{~min}$. A $1.5-\mathrm{mL}$ extract of the supernatant was removed. Two $\mathrm{mL}$ of heptane containing $0.05 \%$ BHT was added to the carrot tissue for a second extraction by the above method. A $1.5-\mathrm{mL}$ extract was removed and combined with the first extract by vortexing for $15 \mathrm{~s}$. An aliquot of $0.5 \mathrm{~mL}$ was filtered through a $0.2 \mu \mathrm{m}$ nylon filter (Fisher Scientific, Pittsburgh) and stored at $-20^{\circ} \mathrm{C}$ until high-performance liquid chromatography (HPLC) analysis. Analysis with $\beta$-Apo- 8 '-carotenal (Fluka, St. Louis) and $\delta$-tocopherol (Matreya, State College, Pa.) internal standards added to lyophilized tissue showed nearly $100 \%$ extraction efficiency, although it is possible that some carotenoids were not extractable due to crystallization.

Samples were analyzed by an HPLC method based upon Okuno et al. (1998). A 50- $\mathrm{\mu L}$ sample was injected into a Supelguard Discovery C18 $(2 \mathrm{~cm} \times 4.0 \mathrm{~mm}, 5 \mu \mathrm{m})$ guard column connected to a Discovery C $18(25 \mathrm{~cm}$ $\times 4.6 \mathrm{~mm}, 5 \mu \mathrm{m}$ ) column (Supelco, Bellefonte, $\mathrm{Pa}$.). The HPLC system consisted of a quaternary pump, a vacuum degasser and a multiple wavelength detector (Agilent 1100 series). The system was controlled by HP ChemStations software (Hewlett-Packard Company, Palo Alto, Calif.). The isocratic mobile phase consisted of methanol at a flow rate of $2 \mathrm{~mL} \cdot \mathrm{min}^{-1}$.

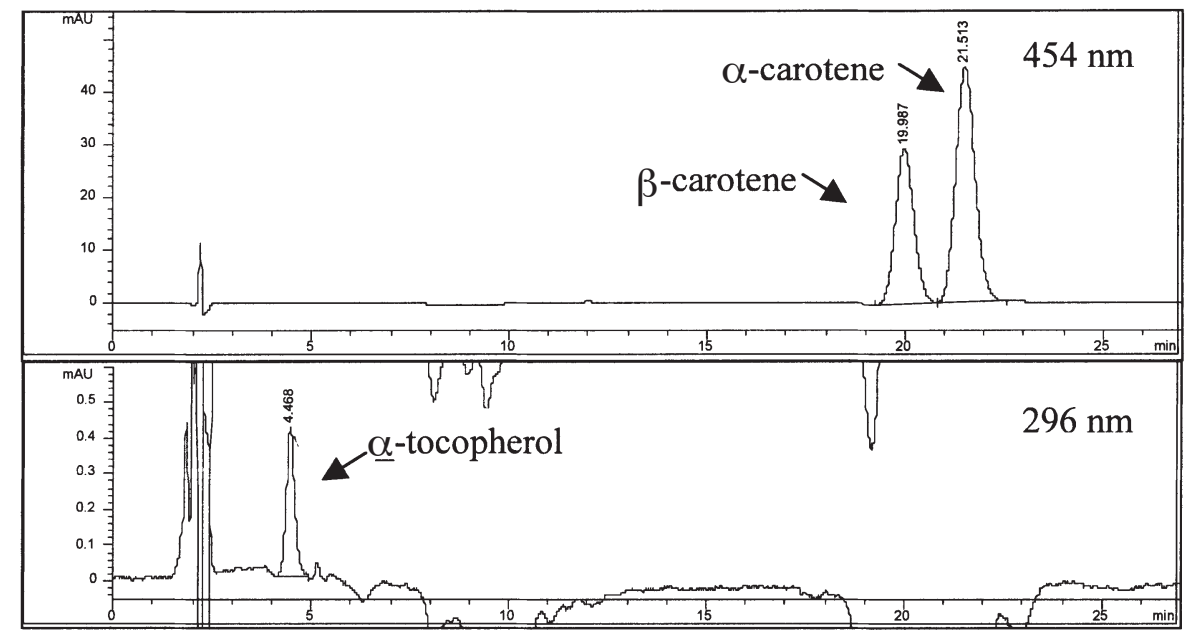

Fig. 1. Typical reversed-phase HPLC chromatography profiles for major carotenoids and tocopherol of commercial fresh market carrot hybrids grown in Randolph, Wisc. in 2001. Lycopene elutes immediately before $\alpha$-carotene ( $454 \mathrm{~nm}$ ) but cannot be seen on this chromatograph due to minimal amount 
Table 1. Cultivar means and standard errors for carotenoids and tocopherols separated by tissue type of commercial fresh market carrot hybrids grown in Randolph, Wisc. in 2001 reported on a dry weight basis.

\begin{tabular}{|c|c|c|c|c|c|c|c|c|c|}
\hline \multirow[b]{2}{*}{ Cultivar } & \multirow[b]{2}{*}{ Source $^{z}$} & \multicolumn{4}{|c|}{ Xylem } & \multicolumn{4}{|c|}{ Phloem } \\
\hline & & lycopene & $\alpha$-carotene & $\beta$-carotene & $\alpha$-tocopherol & lycopene & $\alpha$-carotene & $\beta$-carotene & $\alpha$-tocopherol \\
\hline & & \multicolumn{8}{|c|}{ 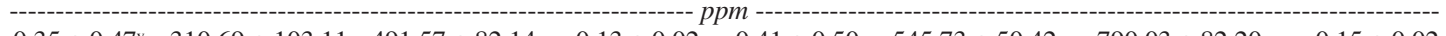 } \\
\hline Sunrise & Crookham & $0.35 \pm 0.47^{y}$ & $310.69 \pm 103.11$ & $491.57 \pm 82.14$ & $0.13 \pm 0.02$ & $0.41 \pm 0.50$ & $545.73 \pm 50.42$ & $790.03 \pm 82.20$ & $0.15 \pm 0.02$ \\
\hline Bolero & Vilmorin & $0.41 \pm 0.38$ & $204.89 \pm 38.26$ & $383.65 \pm 100.34$ & $0.07 \pm 0.02$ & $0.34 \pm 0.47$ & $549.92 \pm 60.86$ & $858.27 \pm 71.77$ & $0.13 \pm 0.01$ \\
\hline Sweet Bites & Harris Moran & $0.73 \pm 0.52$ & $445.72 \pm 46.66$ & $655.41 \pm 35.98$ & $0.14 \pm 0.01$ & $0.31 \pm 0.52$ & $654.35 \pm 47.58$ & $990.78 \pm 107.21$ & $0.12 \pm 0.01$ \\
\hline Nantindo & Harris Moran & $0.30 \pm 0.33$ & $223.52 \pm 16.50$ & $482.33 \pm 25.41$ & $0.13 \pm 0.01$ & $0.00 \pm 0.00$ & $517.14 \pm 69.57$ & $961.69 \pm 104.08$ & $0.14 \pm 0.01$ \\
\hline Bristol & Bejo & $0.46 \pm 0.51$ & $351.34 \pm 22.29$ & $598.16 \pm 111.73$ & $0.08 \pm 0.01$ & $0.26 \pm 0.53$ & $638.73 \pm 39.11$ & $1059.58 \pm 135.14$ & $0.14 \pm 0.02$ \\
\hline Brandon & Bejo & $0.53 \pm 0.46$ & $369.45 \pm 34.32$ & $575.43 \pm 72.93$ & $0.09 \pm 0.01$ & $0.39 \pm 0.31$ & $690.30 \pm 99.82$ & $853.00 \pm 120.51$ & $0.10 \pm 0.02$ \\
\hline Idaho & Bejo & $0.52 \pm 0.04$ & $373.02 \pm 45.15$ & $467.38 \pm 41.33$ & $0.11 \pm 0.02$ & $0.31 \pm 0.11$ & $606.79 \pm 126.59$ & $703.98 \pm 174.86$ & $0.12 \pm 0.02$ \\
\hline \multicolumn{10}{|l|}{ Chantenay } \\
\hline Red Core & Alf Christianson & $0.48 \pm 0.41$ & $251.00 \pm 48.47$ & $392.07 \pm 86.37$ & $0.09 \pm 0.02$ & $0.28 \pm 0.56$ & $506.15 \pm 39.74$ & $894.65 \pm 110.35$ & $0.09 \pm 0.02$ \\
\hline Average & & $0.47 \pm 0.13$ & $316.20 \pm 83.95$ & $505.75 \pm 96.99$ & $0.10 \pm 0.03$ & $0.29 \pm 0.13$ & $588.64 \pm 68.40$ & $889.00 \pm 114.04$ & $0.12 \pm 0.02$ \\
\hline
\end{tabular}

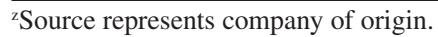

${ }^{y}$ High standard errors for lycopene amounts were due to the extremely low relative amounts of lycopene in orange carrots, which was undetectable in some replicates.

Lycopene, $\alpha$-carotene, and $\beta$-carotene were detected at $454 \mathrm{~nm}$, while $\alpha$-tocopherol was detected at $296 \mathrm{~nm}$. Carotenoid standards were obtained from Sigma (St. Louis). Tocopherol standard was obtained from Matreya.

\section{Results and Discussion}

This method allowed for simultaneous analysis of lycopene, $\alpha$-carotene, $\beta$-carotene, and $\alpha$-tocopherol. Concentrations of carotenoids and tocopherols were calculated on a dry weight basis. Elution times of these compounds (Fig. 1) were lycopene, $22 \min (454 \mathrm{~nm}) ; \alpha$ carotene, $23 \mathrm{~min}(454 \mathrm{~nm}) ; \beta$-carotene, $24 \mathrm{~min}$ $(454 \mathrm{~nm})$; and $\alpha$-tocopherol, $5 \mathrm{~min}(296 \mathrm{~nm})$ Amounts of other tocopherols were unable to be measured due to trace amounts. Average amounts on a dry weight basis (in ppm) were: lycopene, $0.380 \pm 0.157 ; \alpha$-carotene, $452.42 \pm$ 158.95; $\beta$-carotene, $697.37 \pm 222.77$; and $\alpha$-tocopherol, $0.114 \pm 0.025$ (Table 1). Carotenoid values and elution times were consistent with previously published data from carrot (Simon and Wolff, 1987). Tocopherol elution times were consistent with previous data from oat (Tadmor et al., 2000).

This one-pass extraction and HPLC analysis will result in a savings of time by reducing time to analyze both classes of compounds by up to $50 \%$. Material resource needs will also be diminished due to fewer extraction procedures. In the context of a breeding program designed to enhance both carotenoid and tocopherol levels in vegetable crops this can decrease the amount of time between harvest and selection, and thereby increase the efficiency of selection allowing more germplasm to be screened.

\section{Literature Cited}

Buchanan, B.B.,W. Gruissem, and R.L. Jones. 2000. Biochemistry and molecular biology of plants. Amer. Soc. Plant Physiol. Rockville, Md.

Emmons, C.L., D.M. Peterson, and G.L. Paul. 1999. Antioxidant capacity of oat (Avena sativa $\mathrm{L}$.) extracts. II. In vitro antioxidant activity and contents of phenolic and tocol antioxidants. J. Agr. Food Chem. 47:4894-4898.

Grela, E.R., S.K. Jensen, and K. Jakobsen. 1999 Fatty acid composition and content of tocopherols and carotenoids in raw and extruded grass pea (Lathyrus sativus L.). J. Sci. Food Agr. 79:2075-2078.

Grusak, M.A. and D. DellaPenna. 1999. Improving the nutrient composition of plants to enhance human nutrition and health. Annu. Rev. Plant Physiol. Plant Mol. Biol. 50:133-161.

Hirschberg, J. 1999. Production of high-value compounds: carotenoids and vitamin E. Current Opinion Biotechnol. 10:186-191.

Ito, Y., H. Shimizu, T. Yoshimura, R.K.Ross, M.
Kabuto, N. Takatsuka, N. Toku, K. Suzuki, and R. Shinohara. 1999. Serum concentrations of carotenoids, alpha-tocopherol, fatty acids, and lipid peroxides among Japanese in Japan, and Japanese and Caucasians in the US. Intl. J. Vitamin Nutr. Res. 69:385-395.

Jacques, P.F. 1999. The potential preventative effects of vitamins for cataracts and age-related macular degeneration. Intl. J. Vitamin Nutr. Res. 69:198-205.

Okuno, S., M. Yoshimoto, T. Kumagai, and O. Yamakawa. 1998. Contents of $\beta$-carotene and $\alpha$-tocopherol of sweet potato cultivars newly developed for processing purposes. Trop. Agr. (Trinidad) 75:174-176.

Prakash, P., R.M. Russell, and N.I. Krinsky. 2001. In vitro inhibition of proliferation of estrogendependent and estrogen-independent human breast cancer cell treated with carotenoids or retinoids. J. Nutr. 131:1574-1580.

Psomiadou, E., M. Tsimidou, and D. Boskou. 2000. $\alpha$-tocopherol content of Greek virgin olive oils. J. Agr. Food Chem. 48:1770-1775.

Simon, P.W. and X.Y. Wolff. 1987. Carotenes in typical and dark orange carrots. J. Agr. Food Chem. 35:1017-1022.

Tadmor, Y., O. Larkov, A. Meir, M. Minkoff, E. Lastochkin, M. Delestein, S. Levin, J. Wong, T. Rocheford, and E. Lewinsohn. 2000. Reversedphase high performance liquid chromatographic determination of vitamin E components in maize kernels. Phytochemical Anal. 11:370-374. 